\title{
Streaming Video over HTTP with Consistent Quality
}

\author{
Zhi Li \\ Cisco \\ 170 West Tasman Dr. \\ San Jose, CA 95134, USA \\ zhil2@cisco.com \\ Yufeng Shan \\ Cisco \\ 1414 Massachusetts Ave. \\ Boxborough, MA 01719, USA \\ yshan@cisco.com
}

\author{
Ali C. Begen \\ Cisco Canada \\ 181 Bay St., Suite 3400 \\ Toronto, ON M5J 2T3, Canada \\ abegen@cisco.com \\ Bruce Osler \\ Cisco \\ 1414 Massachusetts Ave. \\ Boxborough, MA 01719, USA \\ brosler@cisco.com
}

\author{
Joshua Gahm \\ Cisco \\ 1414 Massachusetts Ave. \\ Boxborough, MA 01719, USA \\ jgahm@cisco.com \\ David Oran \\ Cisco \\ 55 Cambridge Pkwy, Suite 101 \\ Cambridge, MA 02142, USA \\ oran@cisco.com
}

\begin{abstract}
In conventional HTTP-based adaptive streaming (HAS), a video source is encoded at multiple levels of constant bitrate representations, and a client makes its representation selections according to the measured network bandwidth. While greatly simplifying adaptation to the varying network conditions, this strategy is not the best for optimizing the video quality experienced by end users. Quality fluctuation can be reduced if the natural variability of video content is taken into consideration. In this work, we study the design of a client rate adaptation algorithm to yield consistent video quality. We assume that clients have visibility into incoming video within a finite horizon. We also take advantage of the client-side video buffer, by using it as a breathing room for not only network bandwidth variability, but also video bitrate variability. The challenge, however, lies in how to balance these two variabilities to yield consistent video quality without risking a buffer underrun. We propose an optimization solution that uses an online algorithm to adapt the video bitrate step-by-step, while applying dynamic programming at each step. We incorporate our solution into PANDA - a practical rate adaptation algorithm designed for HAS deployment at scale.
\end{abstract}

\section{Categories and Subject Descriptors}

C.2.4 [Computer-Communication Networks]: Distributed applications

\section{General Terms}

Design, Performance

\section{Keywords}

Adaptation, DASH, HTTP, Video, Quality, QoE

\section{INTRODUCTION}

Over the past few years, we have witnessed that streaming video over the Internet is converging towards a new paradigm named HTTP-based adaptive streaming (HAS), also dubbed as dynamic adaptive streaming over HTTP (DASH).

In an HAS system, a video source is chopped into short chunks of a few seconds each (which we will also refer to as segment in this paper). Every segment is independently encoded (or transcoded from a single master high-quality source) at several different bitrates, and the output representations are stored at a server from which clients fetch the segments. Common practice is for the encoder/transcoder to employ constant bitrate (CBR), resulting in a set of tiers, or "levels" of video output. A client application fetches the segments from the server sequentially using plain HTTP GETs, estimates the available bandwidth using measurements of the downloading performance, and adapts the level selection of the next segment to fetch at the completion of the current segment. Typically, tens of seconds of downloaded content are buffered at the client to accommodate bandwidth variability. A viable client rate adaptation algorithm must fetch the video segments to make best use of the available bandwidth, while without risking to drain the client buffer and causing video playout stalls.

The conventional rate adaptation algorithms select the next segment only based on the video bitrate information, but not directly on the video quality of the segment. Thus, during a session, even if the available network bandwidth stays constant, the delivered video quality would vary from a high-motion or high-complexity scene to a low-motion or lowcomplexity scene. For example, in Figure 1 we show two screenshots from a decoded video of an HAS session streamed over a constant-bandwidth link. The first screenshot is from the preview title, which is static and of low complexity. The second one is from a fairly complex and dynamic scene. Not surprisingly, with CBR encoding and bitrate-based adaptation, the second 

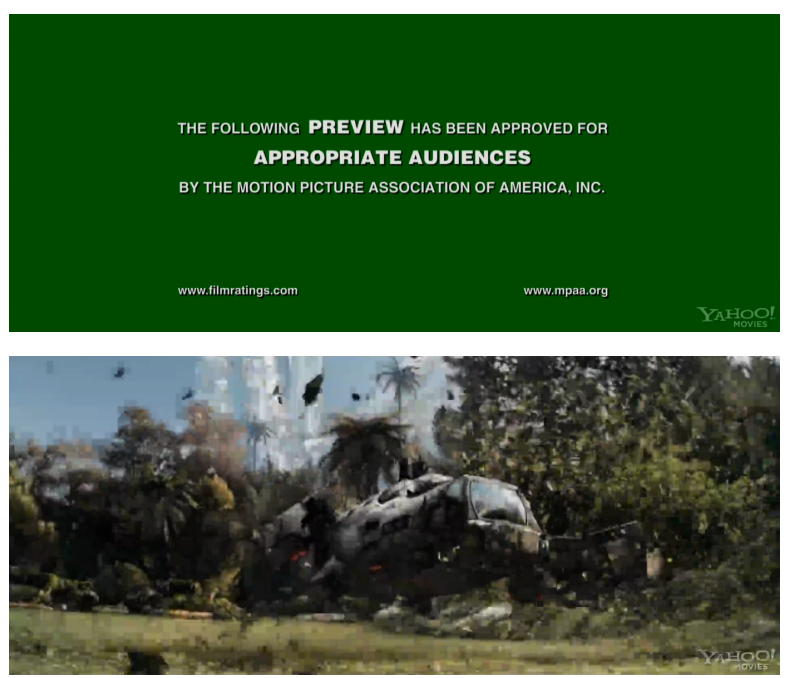

Figure 1: Two screenshots from the decoded video of a HAS session with constant network bandwidth. The video is encoded at multiple CBR levels.

screenshot yields much lower visual quality than the first one.

If we could "steal" some bits from the first scene and "stuff" them into the second one, the overall viewing experience would have been better. With this in mind, everything can boil down to an optimization problem that temporally allocates bits among the video segments to yield an optimal overall quality. This can be considered as the basic rationale behind this work.

We define the optimization objective to be an alphafairness utility function 11, 18 of some quality metric (for example, MSE/PSNR [1], Sarnoff/PQR [4], MSSSIM 20, 21, VQM 16], STRRED 17]), which is generic enough to cover a range of candidates. Heuristically, this objective function could balance between total quality and quality variability.

Besides the bandwidth constraint, the optimization problem is also subject to two other constraints:

- First, the optimization is myopic - it does not know the available bandwidth in the future ${ }^{1}$ and furthermore, in light of live streaming, we assume that the client has visibility into incoming video segments (including both bitrate and quality information) within a finite horizon.

- Second, we make use of the client-side video buffer as a breathing room for video bitrate variability, in a way that the buffer should neither

\footnotetext{
${ }^{1}$ In this work, we do not attempt to introduce a statistical channel model and predict the available bandwidth in the future. This allows our algorithm to be deployed without any assumed knowledge on the network characteristics. Further improvement can be made if a statistical channel model is incorporated if appropriate.
}

be completely drained nor fill above a threshold. If the buffer is completely drained, the playout will stall, which is probably the worst event for an end user's experience. Typically, to also accommodate bandwidth variability, the client buffer size should be bounded above some minimum level (for example, several segments). On the other hand, due to end-to-end latency in live streaming, or device memory limit, or simply economic reasons, the buffer size should also be bounded below some maximum level.

We propose a solution that combines an online algorithm with dynamic programming. The online algorithm adapts the video bitrate step-by-step, and at each step we use dynamic programming to solve a constrained optimization subproblem within a sliding window. The dynamic programming solution allows us to turn a combinatorial problem into something solvable in polynomial time. To our advantage is that, in HAS, as the available bitrate is discrete, it well fits into the dynamic programming framework.

It is worth noting that, our proposed optimization solution should reside in a rate adaptation algorithm at the client side. It is fully orthogonal to the serverside video encoding. For example, in principle, it works with stored video either CBR or variable-bitrate (VBR) encoded at each level. The only needed architectural change is to convey the video quality information to the client in some way (e.g., via the manifest file or an out-of-band approach).

For a sneak preview of what our algorithm is able to achieve, please refer to [2] for some online sample videos.

In the rest of the paper, we first introduce a simple example to illustrate the intuition (Section 2). We then formally state the problem model and formulate the optimization problem (Section 3). We derive the dynamic programming solution for a special case (Section 4), and use it as a building block for the general online algorithm (Section 5). Then, we present how to incorporate the optimization solution into PANDA - a practical client rate adaptation algorithm designed for large-scale HAS deployment (Section 6). Lastly, we present performance evaluation (Section 7) and discuss related work (Section 8).

\section{A SIMPLE EXAMPLE}

Consider the following simple example. Assume that video content of 1 second has already been downloaded and buffered at a client. The client is now trying to decide which video segment to fetch next. It has been given the visibility of the video segments of the current step and one step ahead - it knows the quality and bitrate information of their pre-encoded levels. It also has the information of the current available bandwidth. 


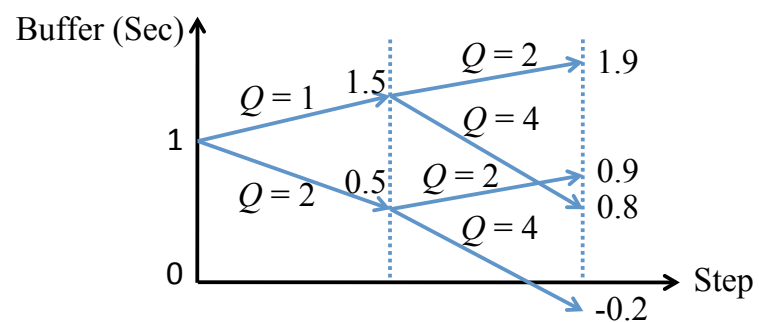

Figure 2: A simple example. A client selects current segment to be fetched, based on information of available bandwidth, buffer size and knowledge of pre-encoded video within a finite horizon of two segments.

If assuming that the bandwidth does not change in the near future, the client can precisely calculate the evolution of the buffer at the end of each step given that a specific segment is fetched.

In this example, at the current step, the client is given two choices - if downloading the low-quality segment, the buffer gain is 0.5 second and the resulting segment quality is 1 ; if downloading the high-quality segment, the buffer loss is 0.5 second and the quality is 2 . Similarly, at the next step, downloading the low-quality segment would result in buffer gain of 0.4 second and segment quality of 2 , and downloading the high-quality segment would result in buffer loss of 0.7 second and segment quality of 4 . Figure 2 illustrates all the possible selections and the resulting position of the client buffer at the end of each step.

Assuming that at the end of the second step, all that matters is that the buffer stays above 0 seconds to avoid video playout stall. The choice $\{h i g h, h i g h\}$ should not be considered because it results in negative buffer of -0.2 second, implying that the video playout will stall. Out of the rest possible choices, if the objective is to maximize the minimum quality out of the two segments, the client should select $\{$ high, low , yielding best minimum quality of 2 . As a result, the client should select the high-quality segment to fetch for the current step. On the other hand, if the objective is to maximize the total quality of the two segments, the client should select $\{l o w, h i g h\}$, yielding best total quality of 5 , and for the current step, the client should select the lowquality segment to fetch. The same procedure repeats at the next step with the new bandwidth and video segment information.

\section{PROBLEM MODEL}

A video stream is chopped into segments $n=$ $1,2,3, \ldots$ of $\tau$ seconds. Each segment has been preencoded at $L$ levels. The resulting quality and bitrate of the $n$-th segment of the $l$-th level are denoted by $Q(n, l)$ and $R(n, l)$, respectively. In conventional CBR encoding, it is assumed that $R(n, l)=R(m, l)$ for $n \neq m$. Our problem model assumes the most general case where $R(n, l)$ at level $l$ can vary from segment to segment.

At each adaptation step $n$, the client selects bitrate $R(n)$ for the current segment to be fetched, from a finite set of available bitrates $\{R(n, l): l=1,2, \ldots, L\}$. The quality corresponding to the selection $R(n)$ is denoted by $Q(n)$.

\subsection{Objectives}

To define the quality optimization objective, we introduce the notion of $\alpha$-fairness [11, 18. For a quality value $q$, its $\alpha$-fairness utility is defined as

$$
U_{\alpha}(q):=\frac{q^{1-\alpha}}{1-\alpha} .
$$

Our optimization objective is to maximize the sum of the $\alpha$-fairness utility over a set of segments:

$$
\max \sum_{n} U_{\alpha}(Q(n))
$$

The rationale is to model resource allocation among a set of segments as a fairness problem. This objective function encompasses a number of special cases. At one extreme, setting $\alpha=0$ corresponds to utilitarianism that accounts for total quality (i.e., "max-sum"). At the other extreme, setting $\alpha \rightarrow \infty$ corresponds to maxmin fairness and minimum quality variability. Setting $\alpha$ between 0 and $\infty$ achieves balance between total quality and quality variability. For example, $\alpha=1$ results in the limiting form $U_{1}(q)=\log q$, and this corresponds to proportional fairness and the Nash bargaining solution [1].

An extension to (1) is to more precisely consider the quality variation by incorporating a multiplier $\delta(n-$ $1, n)$ at each step $n$, and use the following objective function:

$$
\max \sum_{n} \delta(n-1, n) \cdot U_{\alpha}(Q(n)) .
$$

The multiplier $\delta(n-1, n)$ discounts the overall utility if the quality has shifted from one level to another. For example, set $\delta(n-1, n)=1$ if the segments at step $n-1$ and $n$ are selected from the same level, and set $\delta(n-1, n)=0.9$ if they are from different levels. Note that, (2) would be useful if the video source is encoded such that each level corresponds to a constant quality, i.e., $Q(n, l)=Q(m, l)$ for $n \neq m$.

In the following discussions, we assume that the objective function follows the general form $\max \sum_{n} U(n)$ where the utility function $U(n)$ can be either $U_{\alpha}(Q(n))$ or $\delta(n-1, n) \cdot U_{\alpha}(Q(n))$.

\subsection{Constraints}

Let $B(0)$ be the initial buffer size (measured in content seconds), and $B(n)$ the buffer size at the end of 
step $n$. After video playout starts, the buffer evolution can be modeled as

$$
B(n)=B(n-1)+\tau-\tau \cdot R(n) / W(n)
$$

where $W(n)$ is the link bandwidth at step $n$, and $\tau \cdot R(n) / W(n)$ is the segment download duration. That is, in each step, the replenishment of the buffer is $\tau$ seconds, and the depletion of the buffer is $\tau \cdot R(n) / W(n)$ seconds.

The optimization must be subjected to the constraint of client buffer size. Define $B_{L}$ and $B_{H}$ to be the lower and upper buffer bound, respectively, with $0 \leq B_{L} \leq$ $B_{H}$. Except for the initial state where $B(n)<B_{L}$, or for when there is sudden bandwidth variation, the buffer should be maintained such that $B_{L} \leq B(n) \leq B_{H}$. Furthermore, we define a buffer reference level $B_{0}$, towards which the buffer level attempts to converge to.

We note that setting the lower bound $B_{L}$ achieves the balance between the video variability and the bandwidth variability that can be compensated - the higher the $B_{L}$, the more bandwidth variability that can be accommodated, but the less breathing room for video quality variability; vice versa.

Another constraint is the finite horizon $H(n), H(n) \geq$ 1 , in number of adaptation steps (or segments). That is, at step $n$, only the bitrate and quality information of the segments from now to $H(n)-1$ steps ahead, i.e., $\{(R(m, l), Q(m, l)): m=n, n+1, \ldots, n+H(n)-1, l=$ $1,2, \ldots, L\}$, are available to the client's optimization algorithm.

\section{DYNAMIC PROGRAMMING}

In this section, we describe the proposed dynamic programming solution, which is used as a building block in each step of the online algorithm. Within a finite horizon of $H$ steps, given an initial buffer size $B_{\text {init }} \in$ $\left[B_{L}, B_{H}\right]$ and a final buffer size $B_{\text {final }} \in\left[B_{L}, B_{H}\right]$, the dynamic programming algorithm attempts to solve the following problem:

$$
\begin{array}{cl}
\max _{\{R(n)\}} & \sum_{n=1}^{H} U(n), \\
\text { s.t. } & \sum_{n=1}^{H} R(n) \leq H \cdot W, \\
& B(0)=B_{\text {init }}, \\
& B(H)=B_{\text {final }}, \\
& B_{L} \leq B(n) \leq B_{H} \text { for } n=1, \ldots, H,
\end{array}
$$

where $W$ is the available bandwidth, assumed to be constant. The specific value of $B_{\text {final }}$ used will be set in the online algorithm and passed as an input to the dynamic programming algorithm.

How can we solve this problem within polynomial time? Our dynamic programming solution is based on the following intuition. Recall that $R(n)$ is the segment bitrate selected at step $n . R(n)$ has a corresponding utility $U(n)$, which can be evaluated based on (1) or (2). Alternatively, we can write $U(n)$ in terms of the buffer evolution

$$
U((n-1, B(n-1)) \rightarrow(n, B(n)))=U(n),
$$

where the buffer evolves according to (3). The interpretation of (4) is that, by moving the buffer from position $B(n-1)$ at step $n-1$ to $B(n)$ at step $n$, the utility is $U(n)$. Similar utility $U((m, B(m)) \rightarrow$ $(n, B(n)))$ can be defined for the case of $n-m \geq 2$. However, notice that in this case, there may be multiple possible paths to move the buffer from $(m, B(m))$ to $(n, B(n))$, which may result in different utility values. We can then define $U^{*}((m, B(m)) \rightarrow(n, B(n)))$ to be the maximum utility over all the possible paths.

The key to the dynamic programming solution is to realize that the solution to the problem of $H$ steps can be formed using solutions to the problems of lesser steps. First, we show that the following theorem is true:

TheOREm 1. Let $s$ be any intermediate step between two non-adjacent steps $m$ and $n$, or $m<s<n$. It holds true that

$$
\begin{aligned}
& U^{*}((m, B(m)) \rightarrow(n, B(n))) \\
& \max _{B(s) \in\left[B_{L}, B_{H}\right]} U^{*}((m, B(m)) \rightarrow(s, B(s))) \\
& \quad+U^{*}((s, B(s)) \rightarrow(n, B(n))) .
\end{aligned}
$$

Proof. Let $B^{*}(s) \in\left[B_{L}, B_{H}\right]$ be the buffer size at step $s$ that maximizes $U^{*}((m, B(m)) \rightarrow(s, B(s)))+$ $U^{*}((s, B(s)) \rightarrow(n, B(n)))$. For any path from $(m, B(m))$ to $(n, B(n))$, let $B^{\star}(s) \in\left[B_{L}, B_{H}\right]$ be the buffer at $s$ that the path has passed. It holds true that

$$
\begin{gathered}
U((m, B(m)) \rightarrow(n, B(n))) \\
=U\left((m, B(m)) \rightarrow\left(s, B^{\star}(s)\right)\right) \\
\quad+U\left(\left(s, B^{\star}(s)\right) \rightarrow(n, B(n))\right) \\
\leq \quad U^{*}\left((m, B(m)) \rightarrow\left(s, B^{\star}(s)\right)\right) \\
\quad+U^{*}\left(\left(s, B^{\star}(s)\right) \rightarrow(n, B(n))\right) \\
\leq \quad U^{*}\left((m, B(m)) \rightarrow\left(s, B^{*}(s)\right)\right) \\
\quad+U^{*}\left(\left(s, B^{*}(s)\right) \rightarrow(n, B(n))\right)
\end{gathered}
$$

where (5) is by the definition of $U^{*}$ and $(6)$ is by the definition of $B^{*}(s)$. The optimal value is achievable by selecting $B^{\star}(s)=B^{*}(s)$ and recursively selecting the optimal sub-paths.

This theorem states that, going from $(m, B(m))$ to $(n, B(n))$, inevitably one has to pass a mid-way step $s$. At step $s$, one could have many possible buffer sizes $B(s)$. It holds true that, the optimal utility value of a problem $(m, B(m)) \rightarrow(n, B(n))$ has to be the sum of the optimal utility values of the sub-problems $(m, B(m)) \rightarrow(s, B(s))$ and $(s, B(s)) \rightarrow(n, B(n))$ over all possible $B(s)$. So one can solve the problem by solving its sub-problems, by solving its sub-subproblems, and so on. Eventually, things reduce to the baseline case of $(m-1, B(m-1)) \rightarrow(m, B(m))$. 


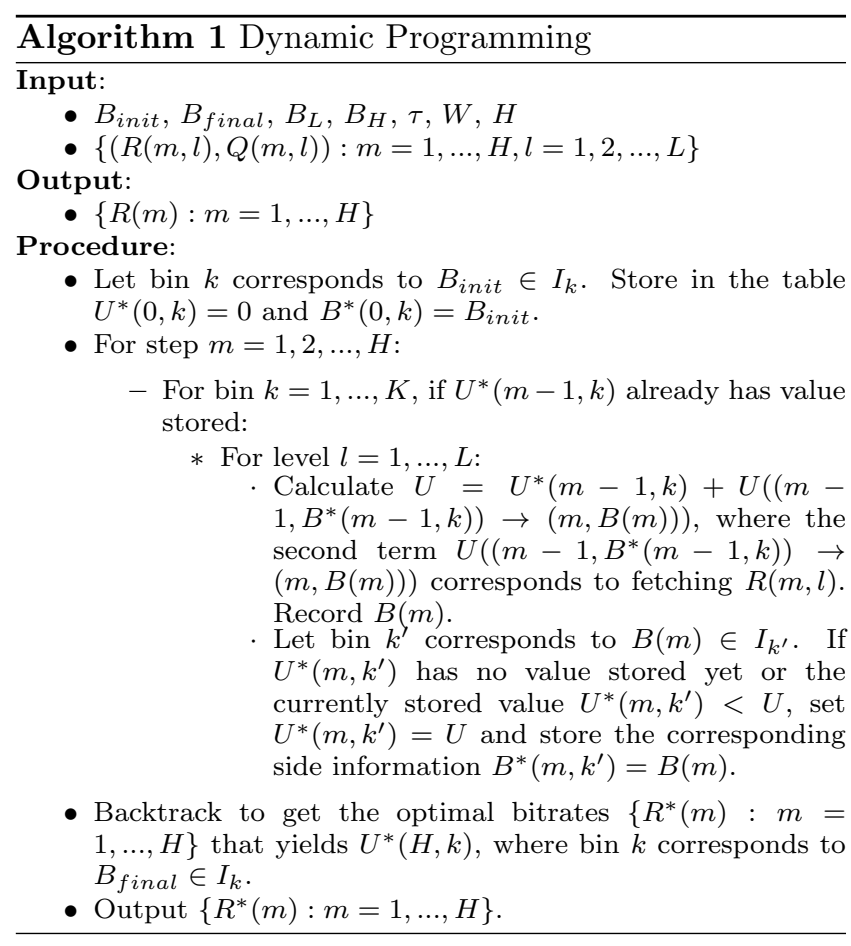

Once a sub-problem has been solved, one can store the solution (including the optimal utility values and some side information for backtracking purpose) in a table for later reuse to save repeated work.

An implementation detail is that, as $\left[B_{L}, B_{H}\right]$ is a continuous interval but the dynamic programming is discrete, we need to quantize $\left[B_{L}, B_{H}\right]$ into $K$ discrete bins with step size $\Delta B$, and only store one optimal utility value for each bin. Denote by $\mathcal{I}:=\left\{I_{1}, I_{2}, \ldots, I_{K}\right\}$ the resulting bins. Thus, the optimal utility values $U^{*}(n, k)$ can be stored in an $(H+1) \times K$ two-dimensional table where the first dimension corresponds to the number of steps (including the initial zeroth step) and the second corresponds to the bins. In the table, we also store the side information $B^{*}(n, k)$, which is the ending buffer size corresponding to $U^{*}(n, k)$.

The dynamic programming solution is described in Algorithm 1. A simple analysis shows that the algorithm has complexity $O(H \cdot K \cdot L)$. In practical implementation in $\mathrm{C}$, we find that with typical parameters (e.g., $H=30, K=50, L=10$ ), the execution time is within a few milli-seconds (e.g., $5 \mathrm{~ms}$ ).

Note that one corner case is, in the second last step of Algorithm 1, we may not find a bin $k$ such that $B_{\text {final }} \in I_{k}$ and there is a value $U^{*}(H, k)$ in it. This may happen if the available bandwidth is either too large or too small for the available pre-encoded video bitrates (recall that we assume no off-intervals between segment downloading in the dynamic programming problem formulation). If this happens, we may find another bin $k^{\prime \prime}$ which has a value $U^{*}\left(H, k^{\prime \prime}\right)$ stored and is closest to $k$, and then perform the backtrace starting from $k^{\prime \prime}$. In this case, we also output the buffer offset

$$
B_{\text {offset }}=B^{*}\left(H, k^{\prime \prime}\right)-B^{*}(H, k)
$$

to be later used in Section 6

\section{ONLINE ALGORITHM}

If the available bandwidth does not vary and the video quality information is available all at once, the dynamic programming algorithm is sufficient to solve the optimization problem in one shot. In reality, the bandwidth changes over time and the video quality information is available within a finite horizon. To deal with this, we propose an online algorithm that repeatedly applies the dynamic programming in a sliding-window manner.

We define a reference buffer level $B_{0}$ that the buffer aims to converge to. We also have a buffer lower bound $B_{L}$ and a buffer upper bound $B_{H}$. However, different from the problem formulation in Section 4 we do not guarantee that the buffer at transient state is bounded within $\left[B_{L}, B_{H}\right]$. For example, when the streaming starts, the initial buffer is zero. Instead, at a particular step $n$, we only make sure that the buffer is bounded within $\left[\min \left(B_{L}, B(n-1)\right), \max \left(B_{H}, B(n-1)\right)\right]$. But over time, thanks to the convergence to $B_{0}$, the buffer is set to be bounded within $\left[B_{L}, B_{H}\right]$ if the bandwidth does not abruptly change.

The finite horizon size at step $n$ is denoted by $H(n)$, which may vary over time in some applications. For example, in live streaming, as the end-to-end latency is bounded, the longer the buffered video is at the client, the shorter the horizon is. Within a window of size $H(n)$, the dynamic programming algorithm is applied, and an optimal rate allocation $\left\{R^{*}(m): m=\right.$ $1, \ldots, H(n)\}$ is obtained. Then the bitrate of the current segment to be fetched is set to be $R^{*}(1)$, i.e., only the most immediate rate is applied.

The online algorithm is described in Algorithm 2

\section{PANDA WITH CONSISTENT QUALITY}

PANDA (reading: Probe-AND-Adapt) is an HAS client rate adaptation algorithm we recently designed to yield high stability and fast responsiveness to bandwidth variations when multiple HAS clients are running within a network domain sharing bottleneck links 12. Performance evaluations show that, compared to conventional HAS algorithms, PANDA is able to reduce the instability of video bitrate selection by over $75 \%$ without increasing the risk of buffer underrun. To detect the available bandwidth, PANDA probes the network by additively incrementing its sending rate at each adaptation step and multiplicatively decreasing its rate if congestion is detected, and adapts its video bitrate accordingly. This "probe and adapt" principle is akin to the additive increase / multiplicative decrease (AIMD) 


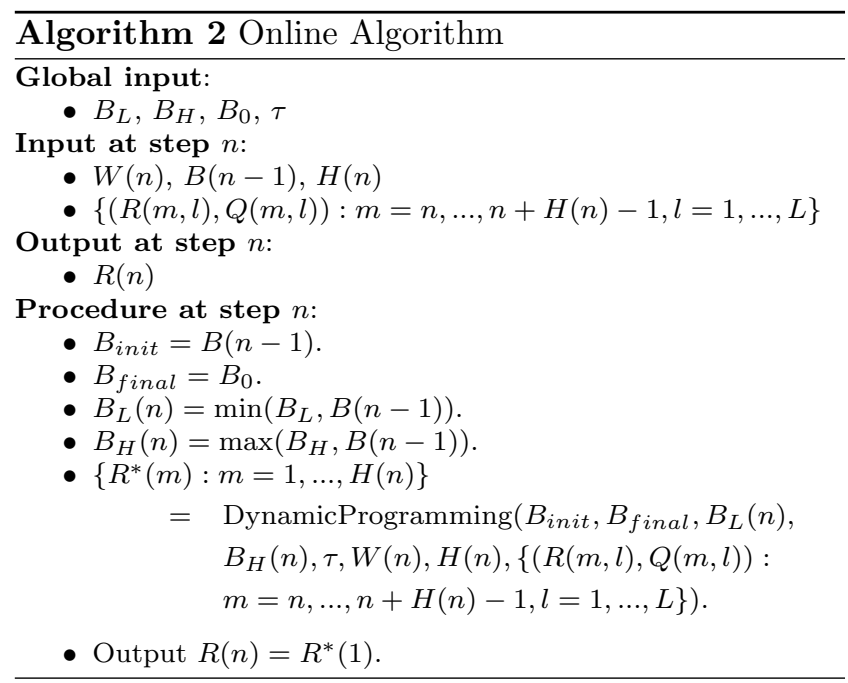

\begin{tabular}{|l|c|c|}
\hline Algorithm & Parameter & Default Value \\
\hline PANDA and PANDA/CQ & $\kappa$ & 0.28 \\
& $w$ & 0.3 \\
& $a$ & 0.2 \\
& $\beta$ & 0.2 \\
& $\tau$ & 2 \\
\hline PANDA only & $B_{0}$ & 20 \\
& $\epsilon$ & 0 \\
\hline PANDA/CQ only & $B_{0}$ & 30 \\
& $B_{L}$ & 10 \\
& $B_{H}$ & 50 \\
& $H$ & 30 \\
\hline
\end{tabular}

Table 1: Default client parameters in ns-2 simulations

principle used in TCP, but it operates in the application layer and at a much longer time scale.

The original PANDA design is video quality-agnostic. In this section, we extend it to incorporate video quality optimization. Our online algorithm naturally fits into PANDA, with the probing part of PANDA providing the bandwidth estimation for the online algorithm, while the online algorithm determining the next segment to fetch and the target inter-request time. We name the new algorithm PANDA with Consistent Quality (PANDA/CQ).

The PANDA/CQ is described in Algorithm 3. For each adaptation step $n$, it proceeds in four sub-steps. In sub-step one, it estimates the available bandwidth using probing, with $k$ and $w$ the two probing parameters controlling the probing convergence rate and the multiplicative decrease threshold, respectively. $T(n)$ is the time duration of step $n$, equal to $\max (\hat{T}(n), \tilde{T}(n))$, where $\tilde{T}(n)$ is the duration for downloading segment $n$. $\tilde{x}(n)$ is the calculated TCP throughput, based on formula $\tilde{x}(n)=R(n) \cdot \tau / \tilde{T}(n)$. The resulting rate $\hat{x}(n)$ is the raw estimation of the bandwidth. In sub-step

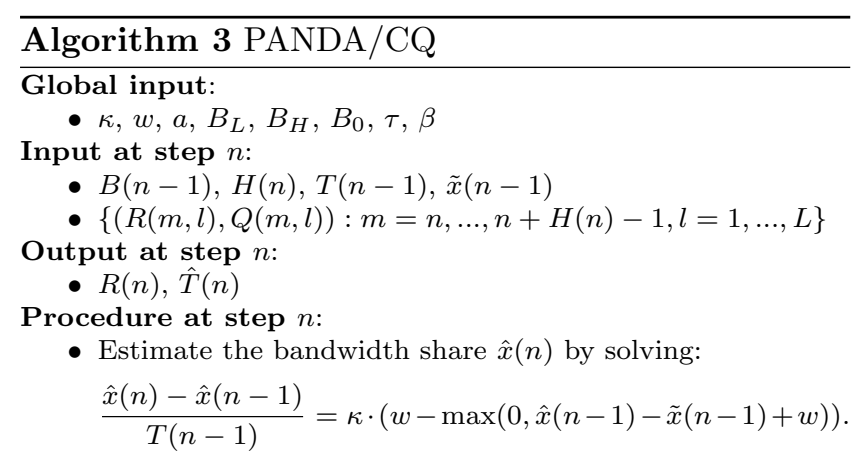

- Smooth out $\hat{x}(n)$ to produce its filtered version $\hat{y}(n)$ by

$$
\frac{\hat{y}(n)-\hat{y}(n-1)}{T(n-1)}=-a \cdot(\hat{y}(n-1)-\hat{x}(n)) .
$$

- Apply the online algorithm to pick the fetched video bitrate $R(n)$ :

$$
\begin{aligned}
R(n)= & \text { Online }\left(B_{L}, B_{H}, B_{0}, \tau, \hat{y}(n), B(n-1), H(n),\right. \\
& \{(R(m, l), Q(m, l)): m=n, \ldots, n+H(n)-1, \\
& l=1, \ldots, L\}) .
\end{aligned}
$$

- Determine the target time until the next request $\hat{T}(n)$ by:

$$
\hat{T}(n)=\frac{R(n) \cdot \tau}{\hat{y}(n)}+\beta \cdot\left(B(n-1)-B_{0}\right) .
$$

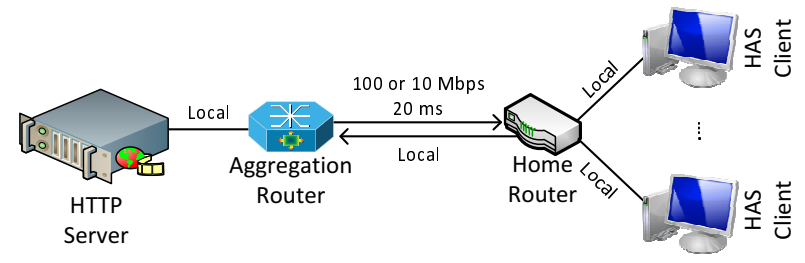

Figure 3: The network topology configured in the ns-2 simulator. Local indicates that the bitrate is effectively unbounded and the link delay is $\mathbf{0} \mathrm{ms}$. 
two, it smoothes out the raw estimation via exponentially weighted moving average (EWMA) filtering, to produce the filtered version of the bandwidth estimation, $\hat{y}(n)$. Here $a$ is a parameter controlling the filtering convergence rate. In sub-step three, $\hat{y}(n)$ is taken as the input available bandwidth (i.e., $W(n))$ of the online algorithm, which generates a video bitrate $R(n)$, to be fetched in the current step. In the last sub-step, it calculates the target inter-fetch interval $\hat{T}(n)$ based on $R(n)$ and $\hat{y}(n)$. $\hat{T}(n)$ also compensates for the current buffer offset $B(n-1)-B_{0}$, with parameter $\beta>0$ controling the convergence speed.

Note that the current form of Algorithm 3 does not handle the corner case of available bandwidth being too large for the available pre-encoded video bitrates. Recall that in Section 4 we discuss that there may be an offset between the target final buffer size $B_{\text {final }}$ and the actual ending buffer size the algorithm produces, assuming no off-intervals between segments. If this offset is positive, we can introduce off-intervals between segment downloads to compensate for the offset (if negative, there is nothing we can do). Thus, to handle this corner case, we can simply replace (8) in the last sub-step with

$\hat{T}(n)=\frac{R(n) \cdot \tau}{\hat{y}(n)}+\beta \cdot\left(B(n-1)-B_{0}\right)+\frac{\max \left(B_{\text {offset }}(n), 0\right)}{H(n)}$

where $B_{\text {offset }}(n)$ is calculated according to $(7)$.

\section{PERFORMANCE EVALUATION}

In this section, we evaluate the performance of the proposed algorithms. The goal is to understand the behavior of individual modules, as well as their aggregate performance. In the first step, we evaluate the dynamic programming solution and the online algorithm in MATLAB simulations. In the second step, we integrate them into the PANDA algorithm and evaluate the performance in the ns- 2 simulator 3 . Besides the simulation results shown in this section, we also provide a few sample videos online [2] for readers' subjective evaluation.

We have identified several existing quality-based HAS schemes $13,9,10,7,8$ (refer to Section 8 for discussions). However, they either focus on a different perspective (e.g., encoding, cross-stream optimization), or are based on different assumptions (e.g., scalable coded video source, statistically stationary source/channel models). Thus, it is not possible to directly compare our scheme with them. Instead, we compare our solution with the bitrate-based adaptation scheme that is unaware of the video quality information.

\subsection{Simulation Setup}

We select two video sources for our evaluation. The first one is a two-minute long 720p Elysium trailer crawled from YouTube 2]. The second one is a twelve-minute long 1080p clip extracted from the movie Avatar. For MATLAB evaluation, we use a two-minute part of the Avatar clip. Each video is chopped into segments of two seconds. The Elysium clip is encoded into seven bitrate levels 400,600, 800, 1200, 1600, 2400 and $3200 \mathrm{Kbps}$, and the Avatar clip in 11 bitrate levels 400, 600, 800, 1200, 1600, 2400, 3200, 4400, 5600, 7000 and 9000 Kbps.

At each level, we use CBR encoding. This is to illustrate that our client algorithm does not require the video to be VBR-encoded, and also for a fair comparison with the bitrate-based adaptation scheme. Note that the proposed algorithm is orthogonal to how the videos are encoded. In practice, we find that (capped) VBRencoded video content generally works better with our client algorithm than CBR.

To measure the video quality, we simply use the negation of mean-squared error (MSE) value for each segment. Note that while it may not be the metric to produce the best visual quality, it is good enough for numerically comparing different schemes. However, in the plots, the MSE values are converted to PSNR for better display, using formula [1:

$$
P S N R=10 \cdot \log _{10}\left(\frac{255^{2}}{M S E}\right) .
$$

In the MATLAB simulation, we input the bitrate and quality information of the segments into the program. Assuming that we know the network bandwidth and there are no gaps between segment downloads, we can precisely calculate the evolution of the client buffer and perform optimization accordingly.

In the ns-2 simulation, we evaluate the scenario of multiple clients sharing a bottleneck link. The network is configured as in Figure 3. The queueing policy used at the aggregation router-home router bottleneck link is the following. For a link bandwidth below or equal to $20 \mathrm{Mbps}$, we use random early detection (RED) with (min_thr, max_thr,p) $=(30,90,0.25)$. The default parameters used in the PANDA/CQ and the original PANDA algorithms are listed in Table 1 .

\subsection{Dynamic Programming}

In the first experiment to evaluate the dynamic programming solution, we compare three schemes: 1) bitrate-based fetching that is unaware of the quality information, 2) dynamic programming that maximizes the minimal quality and 3) dynamic programming that maximizes the mean quality (equivalently, the total quality). We set the lower and upper bounds of the buffer to be loose (20 and 50 seconds, respectively) so that we can see the best gain achievable by quality optimization.

Figure 4 shows the traces of the quality (converted 


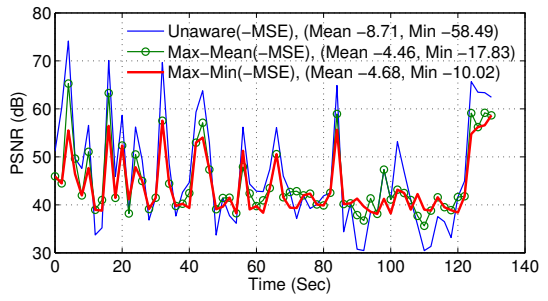

(a1) Quality (Elysium)

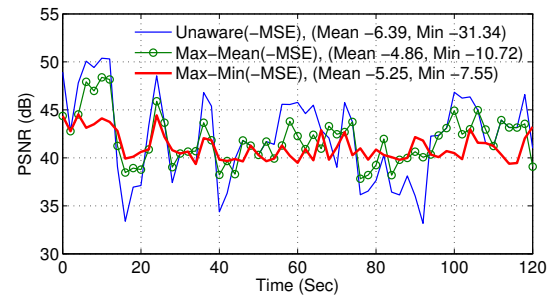

(b1) Quality (Avatar)

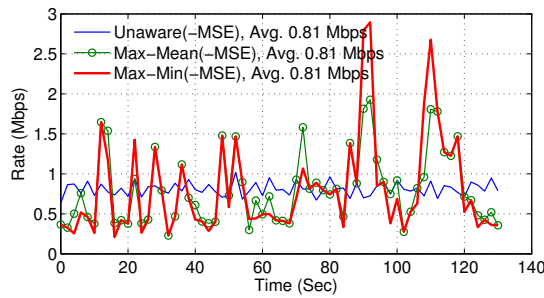

(a2) Rate (Elysium)

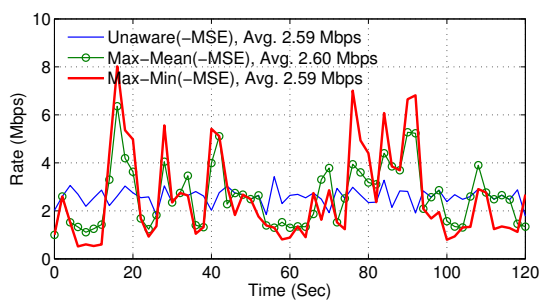

(b2) Rate (Avatar)

Figure 4: Comparing the traces of three schemes: 1) bitrate-based fetching that is unaware of the quality information (Unaware(-MSE)), 2) dynamic programming solution that maximizes the minimal quality (Max-Min(-MSE)) and 3) dynamic programming solution that maximizes the mean quality (Max-Mean(-MSE)). The available bandwidth is set at constant $0.81 \mathrm{Mbps}$ for Elysium and 2.60 Mbps for Avatar. The initial and final buffer levels are 30 seconds; the buffer lower and upper bounds are 20 and 50 seconds, respectively. The reported quality in -MSE is converted to PSNR using (9) for better display.

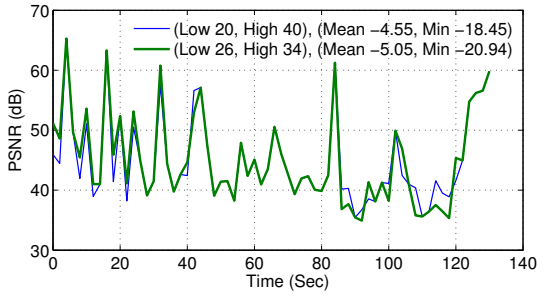

(a1) Quality (Elysium)

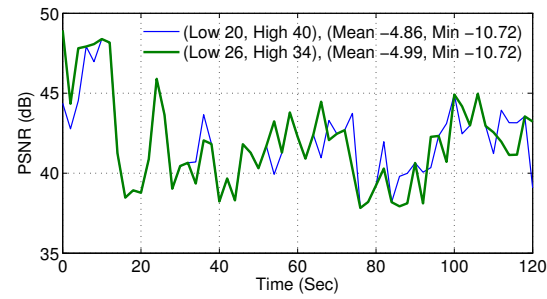

(b1) Quality (Avatar)

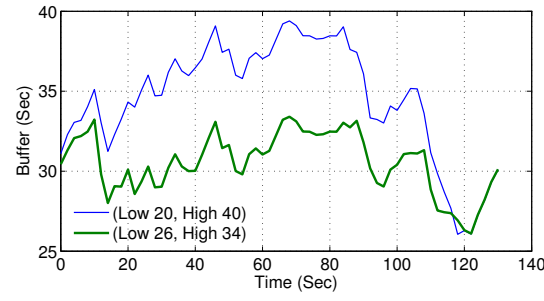

(a2) Buffer (Elysium)

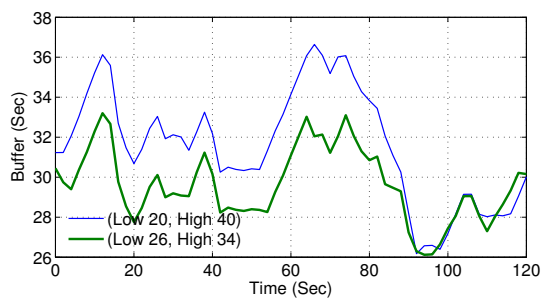

(b2) Buffer (Avatar)

Figure 5: Comparing the traces of dynamic programming solution that maximizes the mean quality with buffer lower and upper bound of 1) 20 and 40 seconds, respectively, and 2) 34 and 40 seconds, respectively. The available bandwidth is set at constant 0.81 Mbps for Elysium and $2.60 \mathrm{Mbps}$ for Avatar. The initial and final buffer levels are 30 seconds. The reported quality in -MSE is converted to PSNR using (9) for better display. 


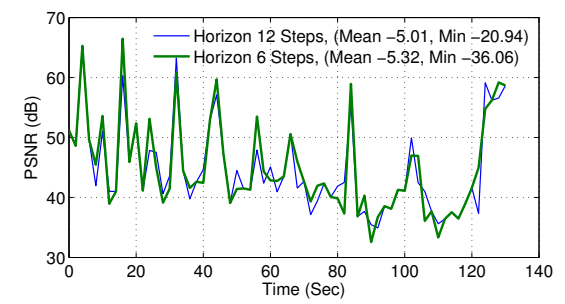

(a1) Quality (Elysium)

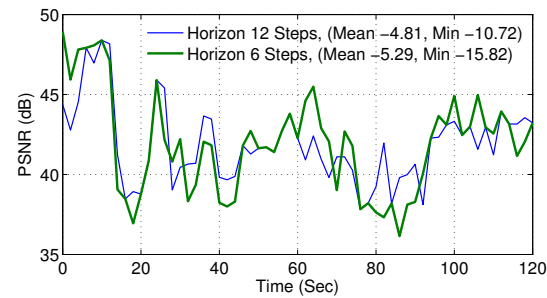

(b1) Quality (Avatar)

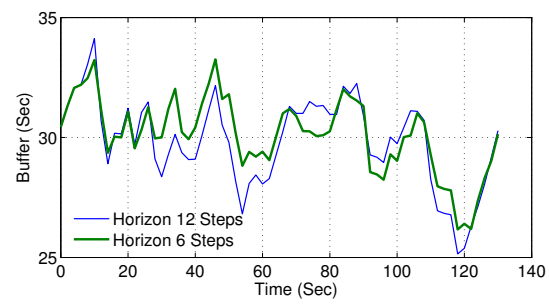

(a2) Buffer (Elysium)

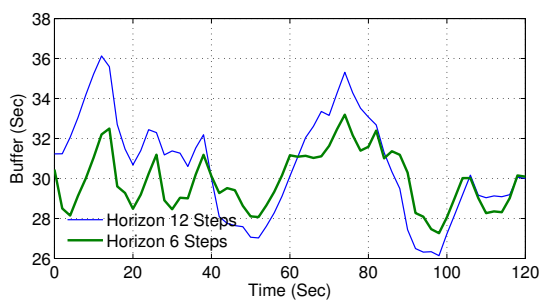

(b2) Buffer (Avatar)

Figure 6: Comparing the traces of online algorithm that maximizes the mean quality with finite horizon of size 1) 12 steps and 2) 6 steps. The available bandwidth is set at constant $0.81 \mathrm{Mbps}$ for Elysium and 2.60 Mbps for Avatar. The initial and final buffer levels are 30 seconds. Buffer lower and upper bounds are 20 and 40 seconds, respectively. The reported quality in -MSE is converted to PSNR using (9) for better display.

to PSNR for better display) and the bitrate of fetched segments for the three schemes. From the quality trace, we can observe that the two quality-optimized schemes yield much better quality than the quality-unaware scheme, both in terms of mean quality and minimal quality. The scheme maximizing the minimal quality achieves best minimum quality (e.g., -10.02 for Elysium compared to -58.49 of the quality-unaware scheme), and the scheme maximizing the mean quality achieves best mean quality (e.g., -4.46 for Elysium compared to -8.71 of the quality-unaware scheme).

We are interested in how the buffer constraint would impact the quality optimization. In the next experiment, we keep the objective to be maximizing the mean quality, and vary the buffer bound in the dynamic programming solution. We test two sets of lower an upper bounds: $\left(B_{L}, B_{H}\right)=(20,40)$ and $\left(B_{L}, B_{H}\right)=$ $(26,34)$ seconds. The reference buffer level $B_{0}$ is set to 30 seconds. The resulting traces of quality, bitrate and buffer evolution are shown in Figure 5. From the buffer evolution plot, we can verify that the resulting buffers are strictly within the specified lower and upper bounds. From the quality trace plot, we can see that the optimal mean quality decreases as the upper bound becomes tighter, which well agrees with our intuition. In Figure 7] we show the trend of how the buffer low and upper bound would affect the mean and minimum quality of the two video sources. As the bound becomes loose, the quality improvement will reach a saturation

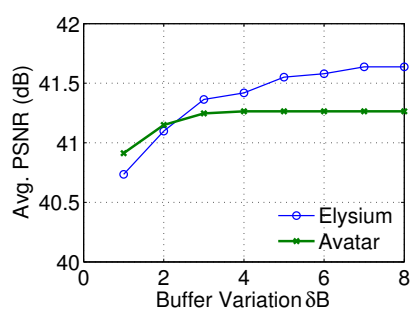

(a) Mean Quality

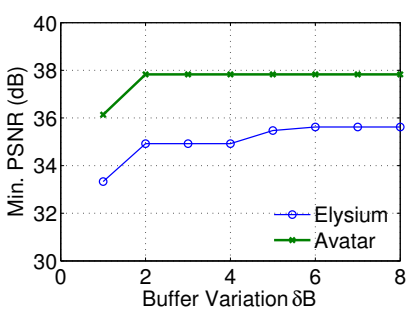

(b) Minimum Quality
Figure 7: The mean and minimum quality as a function of the buffer upper and lower bound $\left(B_{L}, B_{H}\right)=(30-2 \cdot \delta B, 30+2 \cdot \delta B)$, where $\delta B$ varies from 1 to 8 . The initial and final buffer levels are 30 seconds.

point beyond which further loosening the bound would no longer improve the quality.

\subsection{Online Algorithm}

We proceed to evaluate the online algorithm, which uses the dynamic programming solution as a building block. First, we would like to evaluate how the size of the finite horizon would impact the quality optimization result. we keep the objective to be maximizing the mean quality, and compare the finite horizon size of 12 steps and 6 steps. The resulting traces of quality and buffer evolution are shown in Figure 6. From the quality trace plot, we can see that the optimal mean 


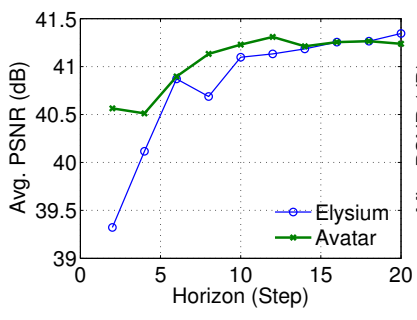

(a) Mean Quality

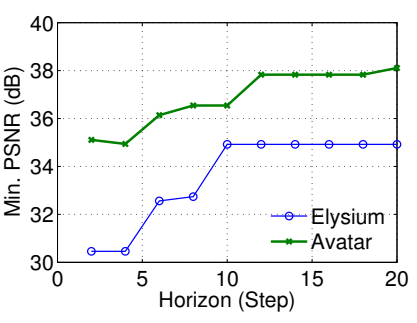

(b) Minimum Quality
Figure 8: The mean and minimum quality as a function of the horizon size from 2 to 20 steps. The initial and final buffer levels are 30 seconds. Buffer lower and upper bounds are 20 and 40 seconds, respectively. The objective is to maximize the mean quality.

quality decreases with a shorter horizon, which well agrees with our intuition that myopic decision yields equal or worse performance. From the buffer evolution plot, it is observed that having a shorter horizon will limit the buffer's variability. This is understood, as being myopic will limit the client to take advantage of the buffer's breathing room. To see the general trend of how the horizon size influences the mean and minimum quality of the two video sources, refer to Figure 8 Note that the non-monotonic behavior may be due to the buffer quantization effect, as discussed in Section 4. Similar to the buffer constraint, there is a similar saturation effect in the horizon constraint, i.e., beyond certain point further improving the horizon would no longer improve the video quality.

\subsection{PANDA/CQ}

Next, we integrate the dynamic programming solution and the online algorithm into the PANDA rate adaptation algorithm, and examine the aggregate behavior of the PANDA/CQ client. Throughout this subsection, we use the objective of maximizing the mean quality. Two things that we are most interested in are: 1) How does the algorithm respond to bandwidth variation? 2) Can the PANDA/CQ client sustain similar stability as the original PANDA?

We first examine the behavior of a single client under variable bandwidth. We compare PANDA/CQ with PANDA under bandwidth variation from 5 Mbps to 2 Mbps and to 5 Mbps (same setting as in [12]). For fairness, we set the multiplicative safety margin $\epsilon$ of PANDA to be 0 , and a lower reference buffer $B_{0}$ of 20 seconds. The resulting traces are compared in Figure 9. From the rate plot, we note that both algorithms are able to closely track the bandwidth variation, thanks to the probing-and-adapt mechanism. PANDA/CQ has a much larger variation of bitrate than PANDA, as its rate adaptation takes into consideration the video content variability. Accordingly, from the buffer plot, the buffer of PANDA/CQ fluctuates within a bounded region; in contrast, the buffer of PANDA stays constant at the reference level.

An important fact to notice from the rate plot is that PANDA/CQ has a higher average fetching bitrate than PANDA (even with $\epsilon=0$ ). The reason behind is that when PANDA/CQ plans on which segment to fetch, it takes into consideration multiple segments in the future. The resulting multiplexing effect creates a more continuous decision space for the PANDA/CQ client to reduce the off-intervals as much as it can. In contrast, in PANDA, the coarse quantization of video bitrate leads to a very discrete decision space, resulting in large off-intervals and low bandwidth utilization. Consequently, from the quality plot, we can see that the mean quality gain for the PANDA/CQ algorithm is higher than the gain noticed in the previous MATLAB simulations, because it is not only contributed by the optimization algorithm, but also the higher bandwidth utilization.

Next, we have multiple clients to compete at a bandwidth-varying link. For each experiment, we have three PANDA or three PANDA/CQ clients compete under bandwidth variation from $5 \mathrm{Mbps}$ to $15 \mathrm{Mbps}$ and to 5 Mbps. Each client start streaming the Avatar video source from a different position. The traces are shown in Figure 10. From the quality plots, we can obviously observe that the PANDA/CQ clients achieves higher mean quality and more stable quality than the PANDA clients. The rate plots show that, similar to the singleclient case, PANDA/CQ clients are able to achieve higher bitrate and more efficient link utilization. Lastly, from the buffer plots, we can see that PANDA/CQ clients have their buffer fluctuate within a bounded region whereas the PANDA clients have their buffers staying constant at the reference level.

Lastly, we keep the link bandwidth to be constant and examine the client behavior as we (a) vary the link bandwidth and (b) vary the buffer lower bound $B_{L}$. We measure the 5-percentile PSNR of all clients's downloaded segments, which considers both total quality and quality variability. The trend plots are shown in Figure 11. From (a), as we increase the link rate, the PANDA/CQ consistently outperforms PANDA by more than $1 \mathrm{~dB}$ on average. Note that the worst-case improvement is much greater (e.g., $5 \mathrm{~dB}$ ). We find that typically it is the worst-case improvement that dominates the perceived visual quality. From (b), the 5-percentile PSNR decreases as we tighten the buffer lower bound, but the minimum buffer increases. Thus, we can see that $B_{L}$ is a parameter that controls the trade off between video quality variability and the risk of buffer underrun. 


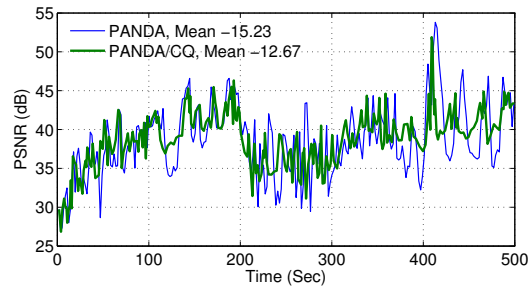

(a) Quality

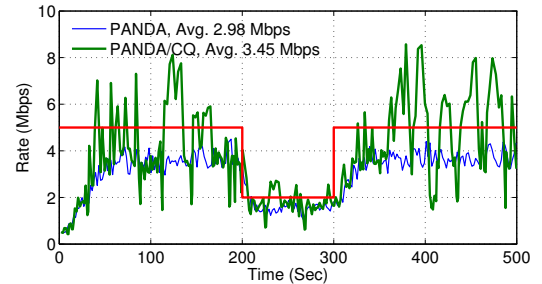

(b) Rate

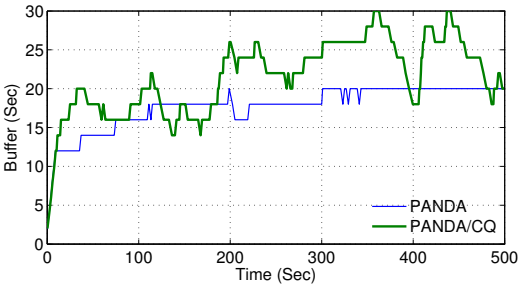

(c) Buffer

Figure 9: Comparing the traces of PANDA/CQ with the original PANDA algorithm for a single client streaming with varying bandwidth. The bandwidth is $5 \mathrm{Mbps}$ for $0^{\sim} 200$ seconds, $2 \mathrm{Mbps}$ for 200 300 seconds and $5 \mathrm{Mbps}$ for 300 500 seconds. Video source: Avatar. The reported quality in -MSE is converted to PSNR using (9) for better display.

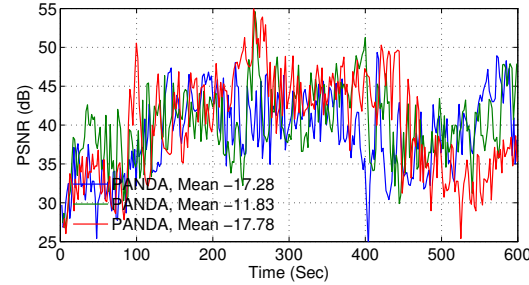

(a1) Quality (PANDA)

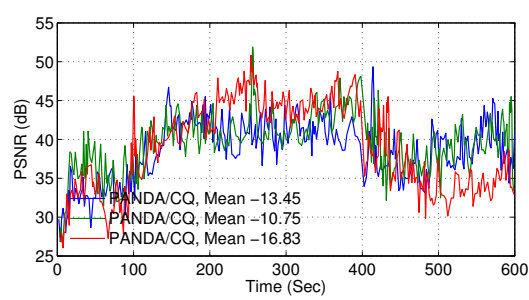

(b1) Quality (PANDA/CQ)

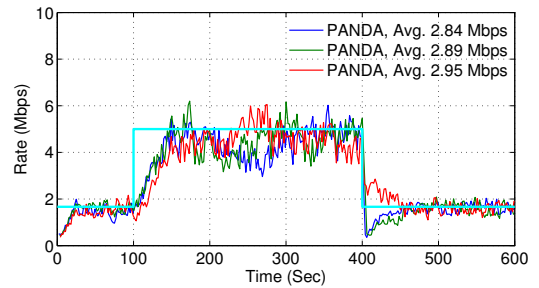

(a2) Rate (PANDA)

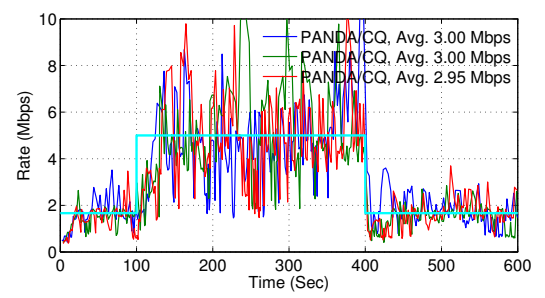

(b2) Rate (PANDA/CQ)

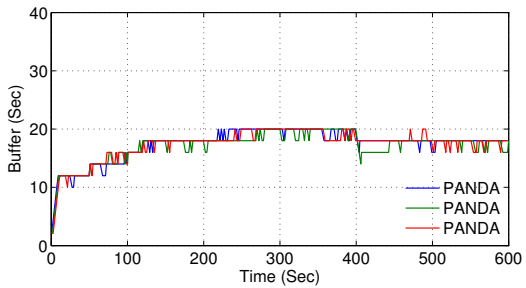

(a3) Buffer (PANDA)

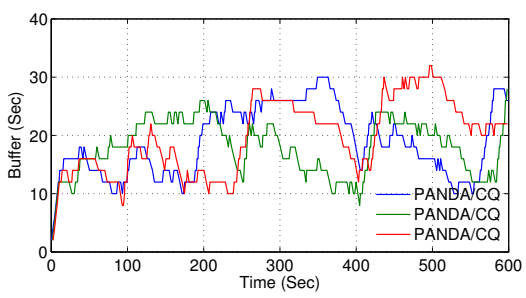

(b3) Buffer (PANDA/CQ)

Figure 10: Comparing the traces of PANDA/CQ with the original PANDA algorithm for three clients sharing a link with varying bandwidth. The bandwidth is 5 Mbps for 0 100 seconds, 15 Mbps for 100 400 seconds and 5 Mbps for 400 600 seconds. Video source: Avatar. Each client starts at a different position of the video clip. The reported quality in -MSE is converted to PSNR using (9) for better display. 


\section{RELATED WORK}

Pre-HAS Video Streaming: The literature on video streaming techniques with quality optimization can be roughly categorized into two eras - the pre-HAS era and the post-HAS era. Early works (e.g., [19) on video streaming assume generic lossy transmission channel. For video streaming over packetized (e.g., IP) networks, before the emergence of HAS, a common wisdom is to lay it on top of lossy RTP/UDP to take advantage of the error-resilient nature of video (e.g., [6]) and apply error control as necessary. Thus, a common theme in these works is to deal with quality degradation caused by packet losses.

Post-HAS Video Streaming: With the emergence of HAS, which rides on top of TCP, packet loss is no longer a concern. Instead, the main source of quality degradation becomes compression and downsampling artifacts. There have been several on-going efforts trying to tackle the video quality optimization problem for HAS, all from different perspectives. Mehrotra and Zhao consider an approach based on rate-distortion optimization and scalable video coding (SVC) 13. They formulate the problem with the buffer constraint in a way similar to ours, and obtain a sub-optimal solution based on Lagrangian multiplier. When attempting to extend their solution from SVC to redundantly encoded multiple rate levels, they have noted that it yields incorrect answer as the rate-distortion curve was not necessarily convex any more. In contrast, our dynamic programming solution does not require convexity in the ratequality relationship.

In 9], a Markov decision process (MDP) is used to compute a set of optimal client strategies in order to maximize the video quality. The MDP requires the knowledge of network conditions and video content statistics, which may not be readily available. Similar statistical and learning-based approaches are proposed by Joseph and de Veciana [10. The optimality of their scheme relies on strong statistical assumptions, such as stationary ergodicity of the source and the channel. In contrast, as explained in the introduction section, we have deliberately avoided a statistical model in this work.

Crabtree et al. report the gains in terms of bitrate saved by using a quality-optimized approach to HAS 7. Their technical discussion mainly focuses on how to assemble a constant quality video stream out of many CBR streams. Georgopoulos et al. study a networkbased approach to ensure the fairness of video quality among HAS streams 8]. The multi-stream problem considered is different from our work, as we focus on quality optimization within a single stream.

There is also some ongoing standardization work in the MPEG. The DASH working group is currently running a core experiment regarding quality-optimized DASH
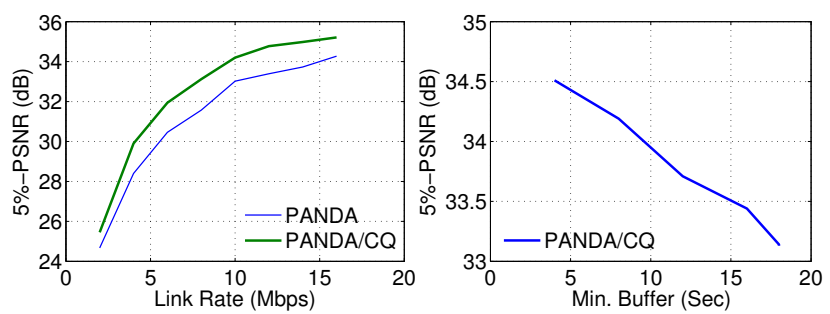

(a) 5\%-PSNR vs. Link Rate

(b) $5 \%$-PSNR vs. Minimum Buffer

Figure 11: Three clients share a constant-bitrate link. (a) 5-percentile PSNR as a function of the link bitrate. (b) 5-percentile PSNR as a function of the resulting minimum buffer, as we vary the buffer lower bound from 4 seconds to 24 seconds.

streaming. The core experiment is still in progress, however, it is expected to result in a signaling approach for carrying quality and/or bitrate information at the segment level.

Video Quality Temporal Pooling: On the study of temporal pooling of video quality, a recent work 15 have shown that the overall impression of a viewer towards a video is greatly influenced by the single most severe event while the duration is neglected, which corroborate our choice of the optimization objective. A more recent study [5] dedicated to temporal pooling for HAS proposes a more complicated linear dynamic system model with the intent to capture the hysteresis effect in human visual response. Joseph and de Veciana [10] uses the difference between mean quality and quality variability as the pooling metric.

Dynamic Programming: Dynamic programming is a combinatorial optimization technique that finds a wide range of engineering applications. The application scenarios we have found that are most related to this work are video encoding for CD-ROMs [14 and quality control for scalable media processing 22 .

\section{CONCLUSION}

In this paper, we have proposed an optimization solution for streaming video over HTTP with consistent quality. We have thoroughly examined the designed algorithms, and integrated it into PANDA - a practical HAS rate adaptation algorithm for HAS deployment at large scale. The proposed solution has the following features:

- It operates independent of whether the source video is CBR or VBR-encoded.

- It is generic enough to cover a range of extant and new video quality models.

- It explicitly takes into account the constraints of bounded client buffer and finite horizon. 
The solution is generic and flexible enough to cover both video-on-demand and live streaming scenarios. Our future work includes building an end-to-end system to deliver video with consistent quality for large-scale HAS deployments.

\section{REFERENCES}

[1] Peak signal-to-noise ratio. Available online: http://en.wikipedia.org/wiki/Peak_signal-tonoise_ratio.

[2] Streaming video over HTTP with consistent quality: Video samples. Available online: https://sites.google.com/site/cqhttpstreaming/.

[3] The network simulator - ns-2. Available online: http://www.isi.edu/nsnam/ns.

[4] Sarnoff JND vision model. White paper available online: http://videoclarity.com/wpcontent/uploads/2013/05/Sarnoff-jnd-1.pdf.

[5] Chao Chen, Lark Kwon Choi, Gustavo de Veciana, Constantine Caramanis, Robert W Heath Jr, and Alan C Bovik. A dynamic system model of time-varying subjective quality of video streams over http. In International Conference on Acoustic, Speech and Signal Processing (ICASSP), 2013.

[6] P.A. Chou and Zhourong Miao. Rate-distortion optimized streaming of packetized media. Multimedia, IEEE Transactions on, 8(2):390-404, 2006.

[7] Barry Crabtree et al. Field trial of quality driven video streaming. In Proceedings of 4 th IEEE International Workshop on Future Multimedia Networking, 2012.

[8] Panagiotis Georgopoulos, Yehia Elkhatib, Matthew Broadbent, $\mathrm{Mu}, \mathrm{Mu}$, and Nicholas Race. Towards network-wide qoe fairness using openflow-assisted adaptive video streaming. 2013.

[9] Dmitri Jarnikov and Tanir Ozcelebi. Client Intelligence for Adaptive Streaming Solutions. EURASIP Journal on Signal Processing: Image Communication, Special Issue on Advances in IPTV Technologies, 26(7):378-389, August 2011.

[10] V. Joseph and G. de Veciana. NOVA: QoE-driven Optimization of DASH-based Video Delivery in Networks. ArXiv e-prints, July 2013.

[11] F. P. Kelly, A. K. Maulloo, and D. K. H. Tan. Rate control for communication networks: Shadow prices, proportional fairness and stability. The Journal of the Operational Research Society, 49(3):237-252, 1998.

[12] Z. Li, X. Zhu, J. Gahm, R. Pan, H. Hu, A. C. Begen, and D. Oran. Probe and Adapt: Rate Adaptation for HTTP Video Streaming At Scale. IEEE Journal on Selected Areas in Communications (J-SAC), Special Issue on
Adaptive Media Streaming, 2014, to appear.

[13] S. Mehrotra and Weidong Zhao. Rate-distortion optimized client side rate control for adaptive media streaming. In Multimedia Signal Processing, 2009. MMSP '09. IEEE International Workshop on, pages 1-6, 2009.

[14] Antonio Ortega, Kannan Ramchandran, and Martin Vetterli. Optimal trellis-based buffered compression and fast approximations. Image Processing, IEEE Transactions on, 3(1):26-40, 1994.

[15] J. Park, K. Seshadrinathan, S. Lee, and A.C. Bovik. Video quality pooling adaptive to perceptual distortion severity. IEEE Transactions on Image Processing, 22(2):610-620, 2013.

[16] Margaret H Pinson and Stephen Wolf. A new standardized method for objectively measuring video quality. Broadcasting, IEEE Transactions on, 50(3):312-322, 2004.

[17] R. Soundararajan and A. C. Bovik. Video quality assessment by reduced reference spatio-temporal entropic differencing. Submitted to IEEE Transactions on Circuits and Systems for Video Technology.

[18] R. Srikant. The Mathematics of Internet Congestion Control. Birkhauser, 2004.

[19] K. Stuhlmuller, N. Farber, M. Link, and B. Girod. Analysis of video transmission over lossy channels. Selected Areas in Communications, IEEE Journal on, 18(6):1012-1032, 2000.

[20] Zhou Wang, Alan C Bovik, Hamid R Sheikh, and Eero P Simoncelli. Image quality assessment: From error visibility to structural similarity. Image Processing, IEEE Transactions on, 13(4):600-612, 2004.

[21] Zhou Wang, Eero P Simoncelli, and Alan C Bovik. Multiscale structural similarity for image quality assessment. In Signals, Systems and Computers, 2003. Conference Record of the Thirty-Seventh Asilomar Conference on, volume 2, pages 1398-1402. IEEE, 2003.

[22] Clemens C Wüst and Wim FJ Verhaegh. Quality control for scalable media processing applications. Journal of Scheduling, 7(2):105-117, 2004. 